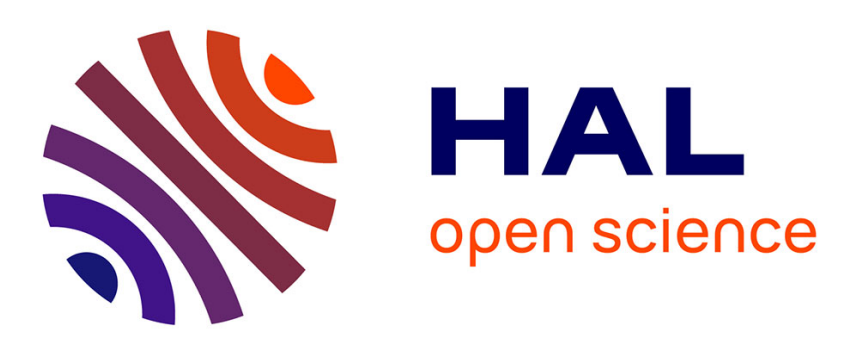

\title{
La parentalité sous contrainte des familles immigrées sans domicile en Île-de-France. Les résultats de l'enquête Enfams
}

Pierre Eloy

\section{- To cite this version:}

Pierre Eloy. La parentalité sous contrainte des familles immigrées sans domicile en Île-de-France. Les résultats de l'enquête Enfams. Revue des politiques sociales et familiales, 2020, Dossier: Parentalités en migration. Normes, pratiques et perceptions, 134, pp.83-92. hal-03081990

\section{HAL Id: hal-03081990 \\ https://hal.science/hal-03081990}

Submitted on 18 Dec 2020

HAL is a multi-disciplinary open access archive for the deposit and dissemination of scientific research documents, whether they are published or not. The documents may come from teaching and research institutions in France or abroad, or from public or private research centers.
L'archive ouverte pluridisciplinaire HAL, est destinée au dépôt et à la diffusion de documents scientifiques de niveau recherche, publiés ou non, émanant des établissements d'enseignement et de recherche français ou étrangers, des laboratoires publics ou privés. 


\title{
La parentalité sous contrainte des familles immigrées sans domicile en île-de-France Les résultats de l'enquête Enfams
}

Pierre Eloy

\author{
Docteur en démographie, Institut de démographie de I’Université de Paris 1, \\ Institut national des études démographiques.
}

\section{La parentalité sous contrainte des familles immigrées sans domicile en île-de-France}

Les résultats de l'enquête Enfams

Depuis la fin des années 1990, le nombre de familles immigrées vivant en hébergement institutionnel a considérablement augmenté. Cela s'explique en partie par l'accroissement des inégalités sociales, la mise en place de politiques migratoires plus restrictives ainsi que les difficultés dans l'accès au logement et à l'emploi. Au regard de ces constats, cet article, qui s'appuie sur les données de l'enquête Enfams (Observatoire du Samusocial de Paris, 2013), analyse les effets de l'absence de logement sur les reconfigurations familiales dans un contexte migratoire. L'article montre qu'à l'éloignement géographique d'une partie des enfants et du conjoint se conjuguent les difficultés du quotidien (accès à l'emploi, garde d'enfant, scolarisation) exacerbées par la précarité administrative, sociale et économique des parents.

Mots-clés : Familles sans domicile - Famille immigrée - Précarité - Hébergement institutionnel - Enquête Enfams

\section{Constrained parenthood in homeless immigrant families in île-de-France}

Results of the Enfams survey

Since the end of the1990s, the number of immigrant families living in institutional accommodation has considerably increased. This is partly explained by widening social inequalities, the setting up of more restrictive migration policies, and more difficult access to housing and work. In this light, this article, based on data from the Enfams survey (Observatoire du Samusocial de Paris, 2013), analyses the effects of homelessness on family reconfiguration in context of migration. The article charts the cumulative effects caused by the geographical separation of some of the children and the spouse, the difficulties of daily living (access to work, childminding, and schooling) and the administrative, social and economic precarity of the parents.

Keywords: Homeless families- Immigrant family - Precarity - Institutional accommodation - Enfams survey. 


\section{L'enquête Enfants et familles sans logement}

L'enquête Enfants et familles sans logement (Enfams) a été réalisée par l'Observatoire du Samusocial en 2013, auprès d'un échantillon de huit cent une personnes vivant en famille et hébergées en île-de-France. Il s'agit de la première enquête quantitative qui a permis $d^{\prime}$ approcher la question de la parentalité en hébergement institutionnel. Les questionnaires, prioritairement administrés aux femmes (en raison de l'intérêt porté aux grossesses et aux expériences de violences), ont été traduits en dix-sept langues et leur passation a été menée en centre d'hébergement d'urgence (Chu), centres d'hébergement et de réinsertion sociale (Chrs), centres d'accueil pour demandeurs d'asile (Cada), et dans des hôtels sociaux. Les objectifs de I'enquête étaient multiples : estimer la taille de la population des familles sans logement en île-de-France, décrire leurs caractéristiques sociodémographiques, caractériser leurs conditions de vie, leur parcours et leur état de santé, ou encore l'impact du sans-abrisme sur la vie quotidienne et sociale des parents et des enfants.

Les personnes non hébergées par les pouvoirs publics au moment de l'enquête n'ont pas été interrogées, tels les hébergés chez un tiers et les personnes dormant dans des lieux non prévus pour l'habitation (tels que la rue) ou encore en bidonville. Cependant, des études ont montré le rôle de l'habitat insalubre dans l'accueil des nouveaux migrants ${ }^{1}$, surtout dans les squats et les bidonvilles ${ }^{2}$. L'enquête Enfams n'est donc pas représentative de l'ensemble des familles sans domicile et sous-estime le nombre d'immigrés en situation de sans-abrisme.

'Simon P., 1996, Les immigrés et le logement : une singularité qui s'atténue, Données sociales, Insee, p. 421-428.

${ }^{2}$ Bouillon F., Dietrich-Ragon P., 2012, Derrière les façades. Ethnographies de squats parisiens, Ethnologie française, vol. 42, n 3, p. 429440. Legros O., 2010, Les pouvoirs publics et les grands « bidonvilles roms " au nord de Paris (Aubervilliers, Saint-Denis, Saint-Ouen), Revue électronique des sciences humaines et sociales, EspacesTemps. net, Textuel, 27 septembre 2010.

Dans les années 1990, la question du sans-abrisme concernait avant tout des hommes seuls et, plus marginalement, des femmes accompagnées d'enfants. À partir des années 2010, la forte augmentation du recours à l'hébergement institutionnel s'associe à une transformation de la structure démographique de la population sans domicile vivant dans les dispositifs de prise en charge, principalement dans les hôtels sociaux (Guyavarch et Garcin, 2014). En effet, le nombre d'adultes sans domicile accompagnés d'au moins un enfant vivant en France dans les agglomérations d'au moins 20000 habitants a augmenté de $58 \%$ entre 2001 et 2012, et atteint désormais 112300 adultes et enfants sans domicile (Mordier, 2016).
Cette évolution s'explique en partie par la forte augmentation du nombre d'immigrés sollicitant les structures d'hébergement institutionnel. En effet, à l'échelle nationale, la progression des adultes sans domicile, immigrés et francophones, est estimée à $+67 \%$ entre 2001 et 2012 et celle des immigrés non francophones à + $207 \%$, soit une population estimée respectivement à 30000 et 15900 personnes en 2012 (Mordier, 2016). Cet accroissement est d'autant plus marqué pour les enfants accompagnant des adultes francophones (+ $125 \%$ ) et non francophones (+ $233 \%$ ). Au total, $62 \%$ des 112300 adultes et enfants sans domicile sont immigrés selon I'enquête Sans-domicile de $2012^{1}$ (Mordier, 2016). Par ailleurs, l'enquête Enfants et familles sans logement (Enfams) (encadré ci-contre) montre que plus de $90 \%$ des 10280 familles hébergées en île-de-France sont immigrées, tout comme les familles ayant recours au numéro d'appel pour les sans-abri, le 115 (Eloy, 2019). La présence immigrée est donc d'autant plus marquée au sein des dispositifs institutionnels accueillant des familles.

Les déterminants du sans-abrisme ont ainsi évolué depuis les premiers travaux quantitatifs réalisés sur cette question (Marpsat, 1999). L'augmentation des flux migratoires, en particulier des demandeurs d'asile depuis la fin des années 1980, cumulée à l'absence de régularisation et aux difficultés importantes que rencontrent les immigrés sur le marché du travail et dans l'accès au logement (Pan Ké Shon et Scodellaro, 2015) ont conduit à la relégation dans les marges d'une frange de plus en plus importante de la population immigrée, notamment en famille.

Si certaines études réalisées auprès de familles hébergées en centre $d^{\prime}$ hébergement et de réinsertion sociale (CHRS) s'intéressent aux effets de la migration sur la parentalité (Costes et Mounir, 2019; Thiery, 2008 et 2010), rares sont les enquêtes quantitatives à explorer cette dimension du sans-abrisme. Dans ce contexte, cet article questionne les spécificités des familles immigrées sans domicile vivant en hébergement institutionnel : quelles sont les raisons ayant conduit ces familles à se trouver sans logement? Et dans quelles mesures l'absence de logement a-t-elle un impact sur I'unité familiale, en particulier dans un contexte migratoire? Pour répondre à ces questions, I'article explore, à l'aide de l'enquête Enfams, le profil sociodémographique des familles immigrées vivant

\footnotetext{
${ }^{1}$ Selon les données rétrospectives de l'enquête Trajectoires et Origines (TeO) de I'Institut national d'études démographiques, réalisée auprès de ménages ordinaires, une part assez faible d'immigrés a été en situation de mal-logement au cours de sa vie. En effet, 11 \% des immigrés déclarent avoir connu une expérience de logement précaire en France, dont moins de 2 \% ont déjà dormi à la rue.
}

Revue des politiques sociales et familiales $n^{\circ} 134-1^{\text {er }}$ trimestre 2020 
en hébergement institutionnel et les formes de précarités cumulatives les affectant. Ces dimensions seront ainsi abordées dans un premier temps. Les contraintes de l'hébergement institutionnel sur l'unité familiale, dans un contexte migratoire, seront ensuite évoquées.

\section{Une configuration familiale fortement liée à l'origine géographique}

\section{Divers profils sociodémographiques}

Alors que les premiers travaux évoquaient une présence plus importante de familles monoparentales dans les années 1990, qui sollicitaient les dispositifs d'hébergement institutionnel suite à une rupture conjugale ou des violences intrafamiliales (Marpsat, 1999), I'augmentation récente de la population sans domicile s'est accompagnée d'un fort accroissement de familles biparentales, désormais majoritaires parmi l'ensemble des familles sans domicile. Cependant, la composition familiale varie fortement selon leur origine géographique. En effet, les familles en provenance d'un pays d'Afrique (hors Maghreb) sont plus souvent composées d'un parent seul (majoritairement la mère) accompagné d'enfant ${ }^{2}$. À l'inverse, les familles immigrées originaires d'un pays de la Communauté des États indépendants (Cei) ${ }^{3}$, ou d'un pays de l'Union européenne (Ue), sont composées à plus de $80 \%$ des deux parents (tableau 1). Par ailleurs, les familles hébergées originaires $d^{\prime}$ un pays d'Afrique déclarent plus souvent un conjoint vivant en dehors du ménage, d'autant plus lorsqu'elles sont originaires d'un pays d'Afrique subsaharienne (23\%). Cette séparation des conjoints se conjugue parfois à l'absence d'un ou plusieurs enfants. Ces situations concernent moins les familles issues d'un pays de la Cei, plus souvent hébergées en couple avec l'ensemble des enfants.

Les familles en provenance de l'Ue (originaires à plus de $90 \%$ de Roumanie et de Bulgarie) ont un profil intermédiaire. Bien que composées des deux parents, elles sont près d'un quart à déclarer avoir un enfant à l'étranger (tableau 1). Ainsi, la différence entre le nombre moyen d'enfants total et le nombre moyen d'enfants vivant en hébergement est particulièrement marquée pour les familles originaires d'un pays d'Afrique subsaharienne ou d'un pays de I'Ue (graphique 1).

En s'intéressant plus spécifiquement aux lieux de vie des enfants ne vivant pas avec leurs parents, les résultats diffèrent nettement selon le type de famille. Les familles monoparentales et les familles biparentales déclarent plus fréquemment que les enfants n'habitant pas avec eux vivent avec d'autres membres de la famille. En outre, lorsque le conjoint vit à l'étranger, les enfants qui ne vivent pas dans le ménage sont plus souvent avec ce dernier. Pour les res-

\section{Définitions}

- Le terme «famille» utilisé dans l'enquête Enfants et familles (Enfams) comprend la présence en hébergement d'un ou des deux parents accompagnés d'au moins un enfant mineur âgé de moins de 13 ans, ou d'une femme (seule ou accompagnée de son conjoint) enceinte de plus de trois mois.

- Selon I'Institut national de la statistique et des études économiques (Insee), le terme «sans domicile» recouvre les personnes vivant à la rue, dans des abris de fortune ${ }^{1}$, ou étant prises en charge par des organismes proposant un hébergement gratuit (ou à faible participation) dans des centres collectifs, des chambres d'hôtel ou des appartements ordinaires ${ }^{2}$.

- Le terme «immigré» fait référence à une personne née étrangère à l'étranger et résidant en France. Une personne est "étrangère " lorsqu'elle déclare une nationalité autre que celle du pays dans lequel elle réside.

- Une personne est dite «sans-papiers» lorsqu'elle ne possède pas les documents d'identité requis dans le pays où elle se trouve et qui est, de ce fait, en situation irrégulière.

- Une personne est en «voie de régularisation" lorsqu'elle est en procédure de demande d'asile, c'est-à-dire qu'elle sollicite une protection internationale hors des frontières de son pays, mais elle n'a pas encore été reconnue comme réfugiée ou bien elle dispose d'un récépissé de demande de carte de séjour.

'Brousse C., 2006, Le réseau d'aide aux sans-domicile : un univers segmenté, Économie et Statistique, n 391, p. 15-34.

Peut-être traduit par "abri précaire ". Sont ainsi distingués les lieux privés (cave voiture cage d'escalier) l'espace public et les lieux fermés (station de métro, gare, centre commercial) ou les lieux ouverts (rue, jardin public, bois) (Brousse C., Rochère B. (de la), Massé E., 2002, Hébergement et distribution de repas chauds : qui sont les sans-domicile usagers de ces services?, Insee Première, ${ }^{\circ} 824$ ).

\footnotetext{
${ }^{2}$ Seulement $4 \%$ des enquêtés sont des hommes. Près de $70 \%$ d'entre eux sont hébergés avec leur conjointe et $23 \%$ sont en situation de monoparentalité. Dans près de $97 \%$ des cas, le chef de ménage des familles monoparentales est une femme.

${ }^{3}$ La Cei regroupe l'Azerbaïdjan, I'Arménie, la Biélorussie, le Kazakhstan, le Kirghizistan, la Moldavie, l'Ouzbékistan, la Russie, le Tadjikistan ou encore le Turkménistan.
} 


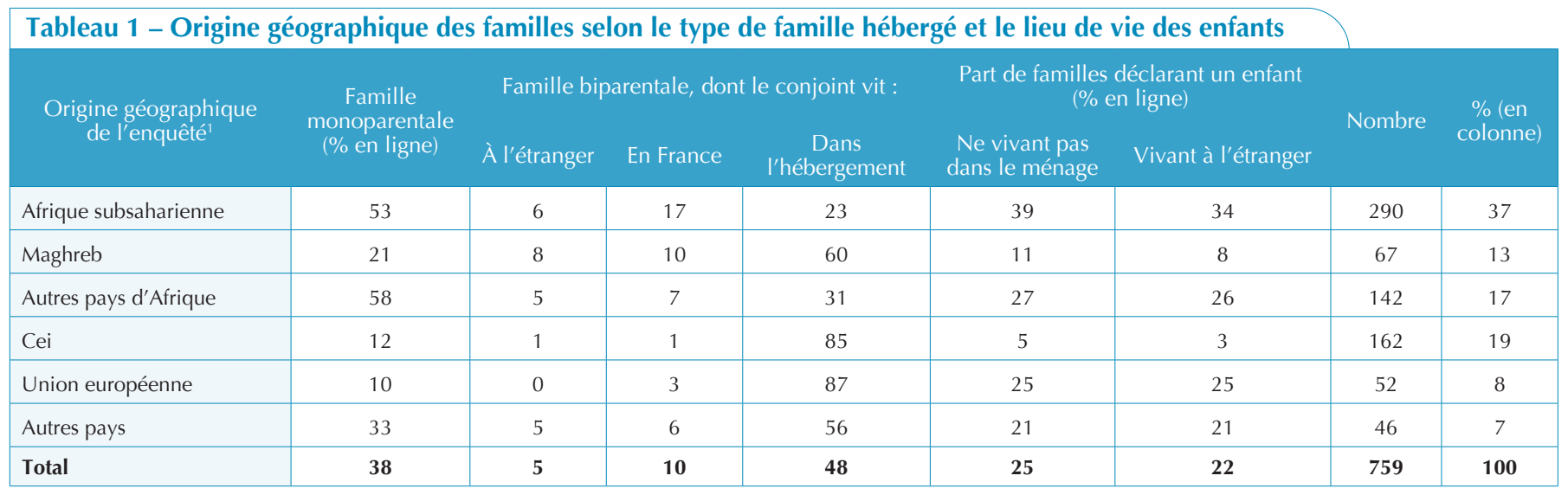

Source : Enquête Enfams, 2013.

Champ : enquêtés immigrés vivant en famille, hébergés $(N=759)$.

Cei : Communauté des Etats indépendants.

Lecture : $39 \%$ des familles originaires d'un pays d'Afrique subsaharienne déclarent un enfant ne vivant pas au sein du ménage.

${ }^{1}$ Les personnes nées en France ne sont pas inclues dans l'analyse.

Graphique 1 - Nombre moyen d'enfants total et vivant dans le ménage selon l'origine géographique

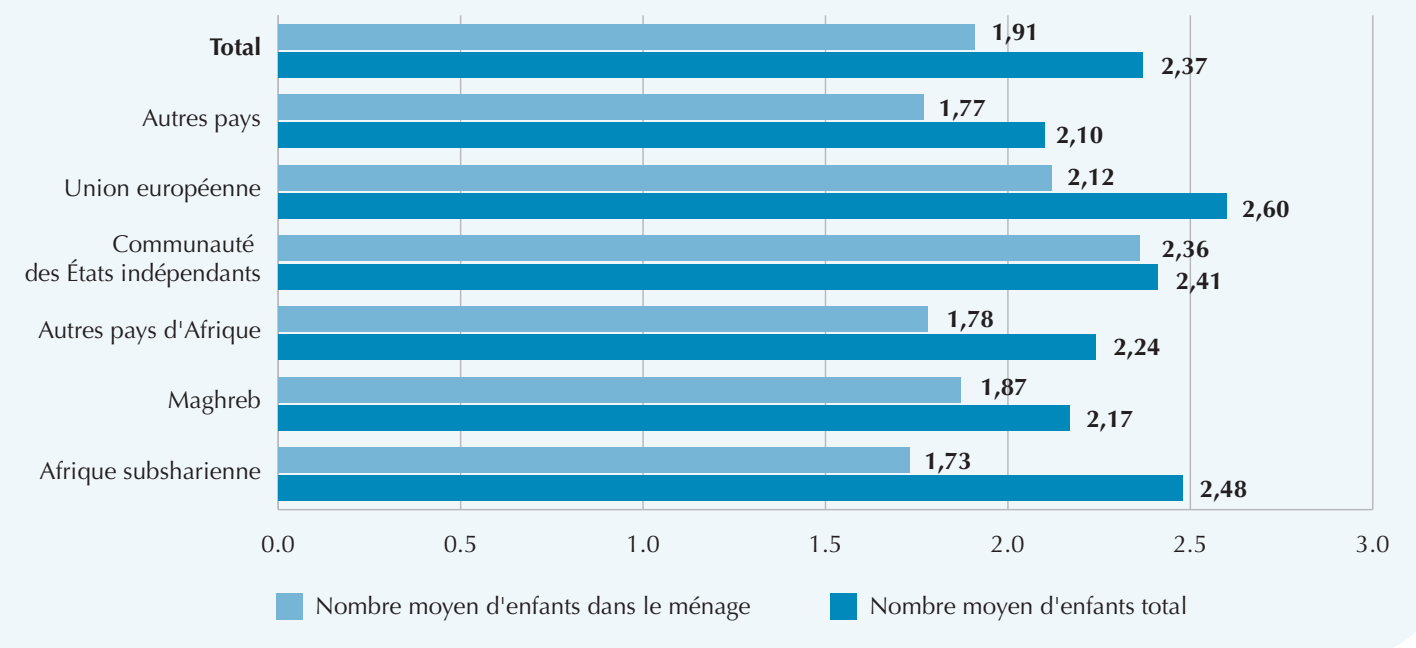

Source : Enquête Enfams, 2013

Champ : enquêtés immigrés vivant en famille, hébergés et déclarant avoir au moins un enfant vivant en dehors du ménage $(\mathrm{N}=211)$.

sortissants de pays africains, il semblerait que la pratique du confiage 4 (Pilon, 2005) soit plus courante, notamment dans l'attente d'une amélioration des conditions de vie.

Les recompositions familiales sont en partie liées aux conditions d'arrivée en France ou aux difficultés rencontrées au cours de la trajectoire migratoire et résidentielle, qui contraignent les familles dans leur projet d'installation (Gosselin et al., 2017).

\section{Des formes de précarité multiples affectant I'unité familiale}

Selon l'ancienneté des flux migratoires, de nombreuses familles peuvent bénéficier de l'aide du réseau de connaissances à leur arrivée (Sayad et Dupuy, 1995). Cette aide se traduit bien souvent par un hébergement chez des tiers (Dietrich-Ragon, 2017). Néanmoins, selon I'origine géographique, le réseau de connaissances est

\footnotetext{
${ }^{4}$ Pratique courante en Afrique qui consiste à confier son enfant à des parents ou amis pendant une période prolongée (Pilon, 2005).
}

Revue des politiques sociales et familiales $n^{\circ} 134-1^{\text {er }}$ trimestre 2020 
Graphique 2 - Lieu de vie des enfants ne vivant pas dans le ménage

Selon le type de famille et le lieu de vie du conjoint

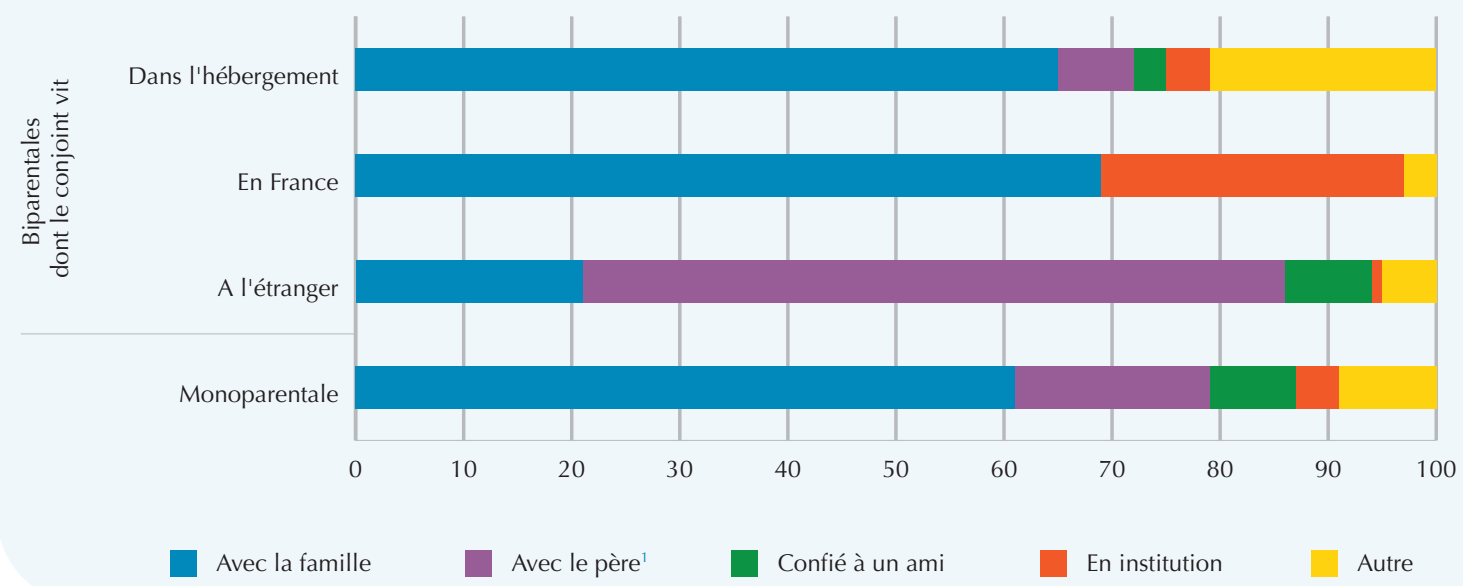

Source: Enquête Enfams, 2013

Champ : enquêtés immigrés vivant en familles, hébergés et déclarant avoir au moins un enfant vivant en dehors du ménage $(N=211)$.

Lecture : au sein des familles biparentales, dont les conjoints vivent dans le même hébergement et dont au moins I'un des enfants vit à l'étranger, $66 \%$ des enfants vivent dans la famille de l'enquêté.

${ }^{1}$ Le père des enfants et le conjoint peuvent être différent.

plus ou moins étendu. Les immigrés sans domicile originaires d'Afrique subsaharienne ou du Maghreb sont plus de $80 \%$ à déclarer connaître au moins une personne en France à leur arrivée, tandis que les immigrés de l'Ue ou de la Cei, dont les migrations sont plus récentes, ne sont que $50 \%$ environ à connaître quelqu'un à leur arrivée (graphique 3). Cependant, l'aide apportée par ces tiers est à nuancer. Parmi les familles déclarant connaître au moins une personne à leur arrivée, seulement $33 \%$ obtiennent beaucoup d'aide de celle-ci. Les familles ayant recours aux dispositifs de prise en charge sont celles qui ne connaissent personne ou qui n'ont pas pu s'appuyer fortement sur la ou les personnes connues.

Graphique 3 - Répartition du nombre de personnes connues à l'arrivée en France

Selon I'origine géographique

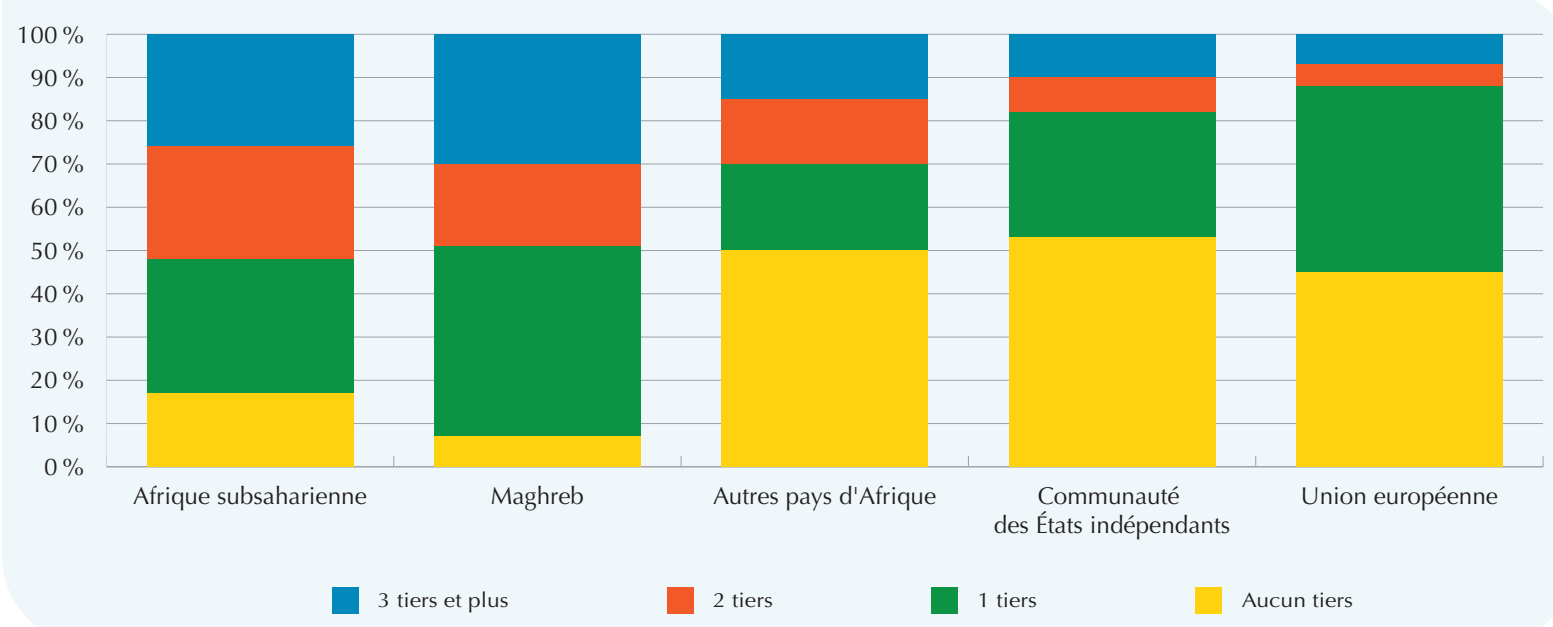

Source : Enquête Enfams, 2013

Champ : enquêtés immigrés vivant en familles, hébergés $(N=759)$.

Revue des politiques sociales et familiales $n^{\circ} 134-1^{\text {er }}$ trimestre 2020

87 Synthèses et statistiques 
Au-delà de l'absence de réseau de connaissances, les familles immigrées sans domicile cumulent d'autres formes de précarité. Bien que les personnes en voie de régularisation représentent environ un quart de la population et que $40 \%$ des familles soient en situation régulière, près $\mathrm{d}^{\prime}$ un tiers n'ont pas de titre de séjour (tableau 2). Les dispositifs d'hébergement d'urgence représentent le dernier filet de sécurité pour ces personnes et l'absence de titre de séjour compromet leur insertion par l'emploi.

En effet, les ressources issues d'un emploi pourraient être un moyen d'améliorer leur condition de vie, toutefois, le taux d'activité dépend en grande partie du statut administratif (tableau 2). Face à l'absence de revenu, seuls les dons perçus par les associations (les tickets restaurant, les colis alimentaires, etc.) permettent d'assurer la survie, grâce aux distributions de produits de première nécessité, en particulier pour les jeunes enfants. À la précarité socioéconomique s'ajoutent les conditions de prise en charge dans les hôtels sociaux [bruit, moisissures, nuisibles, etc. ${ }^{5}$, établissements par ailleurs peu adaptés aux familles nombreuses. En effet, la structure du parc d'hébergement a un impact sur la composition des familles qui peuvent être hébergées : " $5 \%$ des chambres utilisées pour les familles par le 115 de Paris sont pour une seule personne et occupées par des femmes seules, enceintes. Près de la moitié des chambres sont des chambres doubles. Un tiers sont des chambres pour trois.
Au total, seules $15 \%$ des chambres peuvent accueillir au moins quatre personnes. » (Eberhard et al., 2017). Par conséquent, les personnes peuvent être hébergées séparément (pour les familles biparentales), voire être refusées à l'entrée des dispositifs par manque de places adaptées.

Tant les conditions de prise en charge que les différentes formes de précarité posent donc de nombreuses questions quant à leurs effets sur les cellules familiales, les modes d'accueil des jeunes enfants, I'inscription à l'école, les contacts avec les enfants restés au pays et, plus globalement, sur la nature des liens que conservent les parents immigrés sans domicile avec leurs enfants. Autant d'éléments qu'il convient désormais d'aborder.

\section{Vivre en hébergement institutionnel \\ Faire face à de nombreuses difficultés}

Les conditions de la prise en charge en hébergement institutionnel, principalement d'urgence, ont des conséquences dans la recherche d'emploi, notamment en l'absence de modes de garde. En effet, les parents ne peuvent s'absenter en laissant des enfants mineurs dans les hébergements. Ainsi, lorsque les parents vivent avec un enfant âgé de moins de 3 ans $(N=449)$, ils sont $80 \%$ à déclarer n'avoir aucun mode de garde autre que le conjoint, s'il est présent. Près de $13 \%$ des familles ayant un enfant de moins de 3 ans font garder ce dernier dans

Tableau 2 - Situation socioéconomique des familles immigrées sans domicile

\begin{tabular}{|c|c|c|c|c|c|c|c|c|c|}
\hline $\begin{array}{c}\text { Situation } \\
\text { administrative } \\
\text { de l'enquêté }\end{array}$ & Aucune & Rmi-Rsa & $\begin{array}{l}\text { ides sociale } \\
\text { Allocation } \\
\text { chômage }\end{array}$ & $\begin{array}{l}\text { Allocation } \\
\text { familiales }\end{array}$ & $\begin{array}{l}\text { Çues }{ }^{1} \\
\text { Allocation de } \\
\text { rentrée scolaire }\end{array}$ & $\begin{array}{l}\text { Autres } \\
\text { aides }\end{array}$ & $\begin{array}{c}\text { Taux } \\
\text { d'emploi }\end{array}$ & $\begin{array}{l}\text { Revenu médian } \\
\text { (en euro par Uc) }\end{array}$ & $\begin{array}{c}\% \\
\text { (en colonne) }\end{array}$ \\
\hline Régulier & 26 & 37 & 7 & 55 & 13 & 22 & 38 & 464 & 41 \\
\hline En voie de régularisation & 31 & 4 & 5 & 9 & 1 & 14 & 10 & 173 & 26 \\
\hline Sans papiers ${ }^{2}$ & 69 & 1 & 3 & 6 & 1 & 9 & 15 & 15 & 29 \\
\hline Non connu & 2 & 81 & 12 & 80 & 25 & 48 & 38 & 416 & 4 \\
\hline$\%$ en ligne & 39 & 19 & 5 & 29 & 7 & 17 & 24 & 199 & 100 \\
\hline
\end{tabular}

Source: Enquête Enfams, 2013.

Champ : enquêtés immigrés vivant en famille, hébergés en hôtel social, centre d'hébergement d'urgence, centre d'hébergement et de réinsertion sociale ou centre d'accueil pour demandeurs d'asile $(N=759)$.

${ }^{1}$ La somme peut être supérieure à $100 \%$, une famille peut percevoir plusieurs aides différentes.

${ }^{2}$ Les aides déclarées par les personnes sans papiers peuvent être aussi perçues par le conjoint, en situation de régularité.

\footnotetext{
5 Dans l'enquête Enfams, 25 \% des enquêtés déclarent avoir souffert du froid pendant au moins vingt-quatre heures, 34 \% déclarent avoir été confrontés à de graves problèmes d'humidité ou de moisissures et $41 \%$ ont noté la présence de nuisibles dans leur chambre. À cela s'ajoutent les problèmes de nuisance sonore (32\%), de draps de lit en mauvais état (24\%), etc.
}

Revue des politiques sociales et familiales $n^{\circ} 134-1^{\mathrm{er}}$ trimestre 2020 
des crèches, par des assistantes maternelles ou encore dans des haltes-garderies. En outre, I'absence de réseaux de connaissances ou l'éloignement influencent fortement les modes d'accueil : moins de $1 \%$ des parents déclarent avoir recours à un ami ou un parent en plus des modes d'accueil institutionnels. Enfin, près de $6 \%$ de ces familles déclarent disposer d'au moins deux modes de garde possibles.

À ces difficultés de faire garder les plus jeunes enfants, se superposent les difficultés de scolarisation. Parmi l'ensemble des familles, $13 \%$ déclarent avoir au moins un enfant âgé de 3 à 16 ans non scolarisé. Selon Nicolas Oppenchaim et Erwan Le Méner (2015), quatre motifs peuvent être identifiés afin d'expliquer l'absence de scolarisation. Le premier est structurel et concerne $45 \%$ des familles dont au moins l'un des enfants n'est pas scolarisé : la forte mobilité des familles au sein des structures d'hébergement, surtout en urgence, complexifie l'accès à l'école. Les deux motifs suivants découlent de problèmes administratifs et de méconnaissance du système et concernent près de $10 \%$ des parents ayant un enfant non inscrit à l'école. Dans ce cas, les parents déclarent ne pas connaître les démarches, ou ne pas avoir les papiers nécessaires pour l'inscription (attestation de domiciliation, carnet de vaccination à jour, etc.). Le dernier motif est le refus des communes de scolariser les enfants. À ces quatre motifs, très largement interdépendants, un cinquième peut être identifié : la décision des parents de ne pas scolariser leurs enfants âgés de 3 ans ou plus (15\% des familles ayant un enfant non scolarisé). Selon l'enquête Enfams, ce refus s'explique, entre autres, par l'âge des enfants (jugés trop jeunes, bien qu'âgés de plus de 3 ans) ou le fait qu'ils ne soient pas encore propres. De manière plus marginale, cela peut être lié à l'état de santé de l'enfant ou de son parent (handicap physique ou mental nécessitant que l'enfant reste dans la chambre ou qu'il s'occupe de son parent).

Dès lors, les nombreux déménagements, I'absence de place dans les écoles ou encore l'éloignement géographique sont autant de causes structurelles qui recoupent les refus de scolarisation motivés par les communes ou par les parents, qui ne souhaitent pas s'engager dans des démarches longues et fastidieuses. Ces situations sont plus fréquentes lorsque l'accompagnement social est sporadique $(25 \%$ des familles immigrées hébergées ne sont pas suivies par une assistante sociale) ou que les parents ne maîtrisent pas la langue (49 \% d'entre eux ont des difficultés à parler la langue française). En outre, I'absence de scolarisation varie selon le temps passé en France; plus les familles sont arrivées récemment, plus le risque de non-scolarisation est élevé (Oppenchaim et Le Méner, 2015). En lien notamment avec des formes multiples de précarité, les parents peuvent également éprouver des difficultés dans l'exercice de leurs responsabilités parentales (Martin, 2003, p. 36). Des mesures d'accompagnement des pratiques éducatives par des services en milieu ouvert (non renseignées dans l'enquête Enfams), voire dans certains cas des mesures de placements temporaires, peuvent être prises (Eloy, 2019). Parmi l'ensemble des 801 familles enquêtées, seuls treize enfants ont fait l'objet d'un placement, principalement sur motif d'hospitalisation de la mère et donc de l'impossibilité de faire garder les enfants. Ces mesures de placement en institution sont marginales par rapport au nombre d'enfants confiés à des tiers, en particulier dans le pays d'origine.

\section{Maintenir des liens à distance malgré la précarité}

La famille transnationale implique «le maintien de liens des membres d'un groupe de parenté à travers la frontière»(Le Gall, 2005, p.34). Les études sur les familles transnationales montrent le maintien de liens étroits malgré la distance, grâce à la circulation des informations, des biens et des services (ibid.) et accordent une attention particulière à l'analyse des efforts déployés par les personnes pour préserver et renforcer la cohésion familiale. Tout comme d'autres familles immigrées, celles vivant en hébergement institutionnel gardent des liens étroits avec les enfants ne vivant pas dans le ménage. Ainsi, près de $90 \%$ des parents ayant un enfant ne vivant pas dans le ménage déclarent avoir des contacts avec ce dernier.

Cependant, bien qu'il ne soit pas interdit aux personnes hébergées de s'absenter, voire de sortir du pays lorsque leur titre de séjour le permet, l'autorisation de sortie dépend du gestionnaire et du type de structure d’hébergement. Lorsque ce dernier n'est pas averti, les allers et les retours dans le pays d'origine peuvent être un motif d'arrêt de la prise en charge. Les voyages, qui permettent de maintenir les liens avec la famille (Baldassar, 2001), notamment lors des fêtes (Mason, 2004), sont donc rendus plus complexes en raison du coût du voyage et des risques de rupture d'hébergement en cas de départ. Ces contraintes peuvent être nuancées selon les familles. La proximité du pays d'origine, la détention d'un passeport européen ou d'un visa, ainsi que le type de transport utilisé peuvent pallier l'absence de ressources. Les ressortissants d'un pays de la Cei ou de l'Ue déclarent

Revue des politiques sociales et familiales $n^{\circ} 134-1^{\text {er }}$ trimestre 2020 


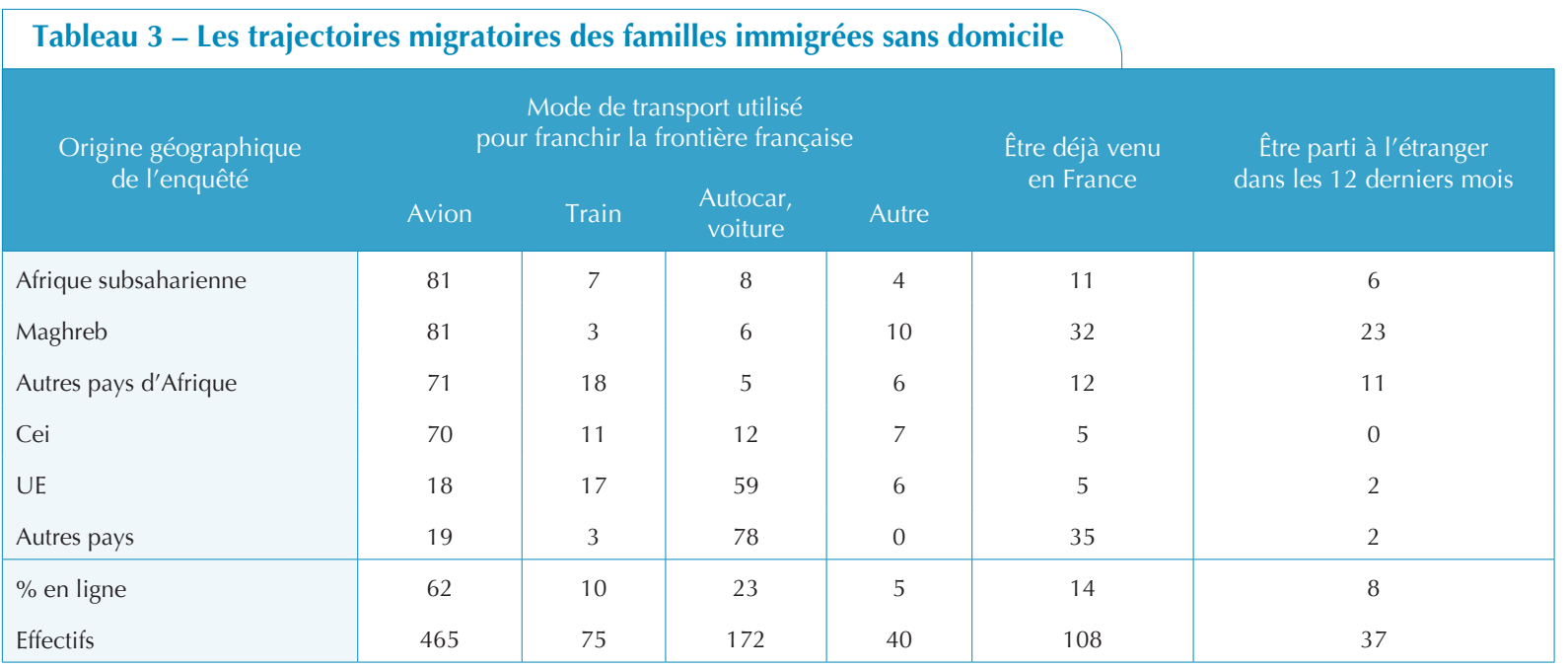

Source : Enquête Enfams, 2013.

Champ : enquêtés immigrés vivant en famille, hébergés et déclarant avoir au moins un enfant vivant en dehors du ménage $(N=759)$.

Cei : Communauté des Ettats indépendants ; UE : Union européenne.

Lecture : $11 \%$ des familles originaires d'un pays d'Afrique subsaharienne déclarent être déjà venues en France au moins une fois avant l'enquête.

majoritairement avoir traversé la frontière française en voiture ou en autocar, à l'inverse des ressortissants africains qui prennent plus souvent l'avion (tableau 3). Les transports par voie terrestre (voiture, autocar) sont moins coûteux et les familles originaires de I'Ue bénéficient également $d^{\prime}$ une facilité de circulation tout comme celles qui ont un visa. Ainsi, les ressortissants d'un pays de l'Ue et d'un pays du Maghreb sont près d'un tiers à être déjà venus en France, et certains déclarent avoir voyagé à l'étranger au cours des douze derniers mois (tableau 3). Les allers et retours entre la France et le pays d'origine sont majoritairement motivés par des visites à la famille et aux amis. Ces déplacements ne concernent qu'une partie marginale des immigrés hébergés. En effet, les familles d'Afrique subsaharienne se trouvent doublement pénalisées sur cet aspect : I'absence de titre de séjour se conjugue à l'éloignement géographique du pays et donc à l'impossibilité de rendre visite à la famille à cause de la faiblesse de leurs revenus.

Les études récentes ont montré que, malgré la distance et l'impossibilité de voyager, les familles transnationales s'efforcent d'assumer leurs responsabilités, notamment à travers des transferts monétaires (Gall et Meintel, 2011). Les flux monétaires sont en grande partie liés au niveau de ressources économiques et à la présence d'un enfant à l'étranger. Cependant, seule une minorité de familles sans domicile (33\%) peut se permettre d'envoyer de I'argent au pays.

\section{Conclusion}

Les familles immigrées sans domicile et vivant en hébergement institutionnel font face à de nombreuses difficultés. L'absence de titre de séjour, les discriminations notamment dans l'accès aux soins (Perret et al., 2019) ou encore la faiblesse des ressources et en particulier l'absence de réseau de connaissances viennent accentuer leur niveau de précarité. Bien que les contacts soient réguliers (physiques pour ceux qui sont en France, par téléphone, appels vidéo, etc. pour les autres parents), ces femmes et ces hommes ne peuvent contribuer pleinement à l'éducation des enfants ne vivant pas avec eux. Les conditions de vie complexifient le rapport qu'entretiennent les parents avec leurs enfants, d'autant plus lorsque ces derniers se trouvent à l'étranger. En outre, ces contraintes posent de nombreuses questions quant aux conséquences dans la trajectoire des enfants ayant grandi en hébergement (Guyavarch et al., 2016 ; Macchi et Oppenchaim, 2019), d'autant plus au regard de la quasi-absence d'actions de soutien à la parentalité dans les structures de prise en charge, surtout en centre d'accueil de demandeurs d'asile et en hôtel social. Autant d'éléments qui viennent mettre à mal les rôles parentaux et renforcer les difficultés quotidiennes rencontrées par les enfants sans domicile et dont les effets ont été largement inexplorés dans les enquêtes.

Revue des politiques sociales et familiales $n^{\circ} 134-1^{\text {er }}$ trimestre 2020 
Baldassar L., 2001, Visits home: Migration experiences between Italy and Australia, Melbourne, Melbourne University Press.

Bouillon F., Dietrich-Ragon P., 2012, Derrière les façades. Ethnographies de squats parisiens, Ethnologie française, vol. $42, n^{\circ} 3$, p. $429-440$.

Brousse C., 2006, Le réseau d'aide aux sans-domicile : un univers segmenté, Économie et Statistique, n 391, p. 15-34.

Brousse C., Rochère B. (de la), Massé E., 2002, Hébergement et distribution de repas chauds, qui sont les sansdomicile usagers de ces services, Insee Première, $\mathrm{n}^{\circ} 824$.

Costes L., Mounir H., 2019, La petite enfance issue de l'immigration à l'épreuve du centre maternel : la « fabrique de la relation mère-enfant », Migrations Société, vol. 4, n 178, p. 55-68.

Dietrich-Ragon P., 2017, Aux portes de la société française. Les personnes privées de logement issues de l'immigration, Population, vol. $72, \mathrm{n}^{\circ} 1$, p. 7-38.

Eberhard M., Guyavarch E., Le Méner E., 2017, Structure familiale et hébergement d'urgence au 115 de Paris, Revue des politiques sociales et familiales, n 123, p. 123-129.

Eloy P., 2019, Héberger les vulnérables, éloigner les indésirables. Trajectoires de prise en charge des familles immigrées sans abri à Paris, thèse de doctorat en démographie sous la direction de Patrick Simon, Université Paris 1 - PanthéonSorbonne.

Gall J.-L., Meintel D., 2011, Liens transnationaux et transmission intergénérationnelle : le cas des familles mixtes au Québec, Autrepart, vol. 1, n 57-58, p. 127-143.

Gosselin A., Desgrées du Loû A.-D., Lelièvre É., 2017, L'installation en France au fil des décennies : la situation a-t-elle évolué ?, in Desgrées du Loû A.-D. (dir.) Parcours. Parcours de vie et santé des Africains immigrés en France, Paris, La Découverte, p. 52-72.

Guyavarch E., Garcin E., 2014, Publics hébergés par le 115 de Paris : une forte progression des familles, Informations sociales, $\mathrm{n}^{\circ} 182$, p. 142-149.

Guyavarch E., Le Méner E., Oppenchaim N., 2016, La difficile articulation entre les espaces du quotidien chez les enfants sans logement, Les Annales de la recherche urbaine, $\mathrm{n}^{\circ} 111$, p. 18-29.

Le Gall J., 2005, Familles transnationales : bilan des recherches et nouvelles perspectives, Les Cahiers du Gres, vol. 5, $\mathrm{n}^{\circ} 1$, p. 29-42.

Legros O., 2010, Les pouvoirs publics et les grands « bidonvilles roms » au nord de Paris (Aubervilliers, Saint-Denis, Saint-Ouen), Revue électronique des sciences humaines et sociales, EspacesTemps.net, Textuel, 27 septembre 2010.

Macchi O., Oppenchaim N., 2019, Grandir dans une chambre d’hôtel, Agora débats/jeunesses, nº 83, p. 111-124.

Revue des politiques sociales et familiales $\mid n^{\circ} 134-1^{\text {er }}$ trimestre 2020

91 Synthèses et statistiques 
Marpsat M., 1999, Un avantage sous contrainte : le risque moindre pour les femmes de se trouver sans abri, Population, vol. $54, n^{\circ} 6$, p. 885-932.

Martin C., 2003, La parentalité en question. Perspectives sociologiques, rapport au Haut Conseil de la population et de la famille : https://halshs.archives-ouvertes.fr/halshs-00201825 (consulté le 17 décembre 2019).

Mason J., 2004, Managing kinship over long distances: The significance of 'the visit', Social Policy and Society, vol. 3, $\mathrm{n}^{\circ} 4$, p. $421-429$.

Mordier B., 2016, Introduction de cadrage : les sans-domicile en France : caractéristiques et principales évolutions entre 2001 et 2012, Économie et Statistique, n 488-489.

Oppenchaim N., Le Méner E., 2015, L'attachement à l'école des familles sans-logement à l'épreuve de l'instabilité résidentielle, Métropolitiques. https://www.metropolitiques.eu/L-attachement-a-I-ecole-des.html (consulté le 17 décembre 2019).

Pan Ké Shon J.-L., Scodellaro C., 2015, L'habitat des immigrés et des descendants : ségrégation et discriminations perçues, in Beauchemin C., Hamel C., Simon P. (dir.) Trajectoires et origines : enquête sur la diversité des populations en France, Paris, Ined éditions, collection Grandes enquêtes, p. 471-497.

Perret G., Vandentorren S., Chauvin P., Vignier N., Vuillermoz C., 2019, Les refus de soins à cause de la CMU ou de l'AME rapportés par les femmes en famille, sans domicile, hébergées en Île-de-France, Bulletin épidémiologique hebdomadaire, $\mathrm{n}^{\circ} 17-18$, p. 341-350.

Pilon M., 2005, Confiage et scolarisation en Afrique de l'Ouest : éclairages à partir des sources de données démographiques, Congrès de I'Union internationale pour l'étude scientifique de la population, Séance ${ }^{\circ} 751$ - Scolarisation et Changements Démographiques/ https://www.documentation.ird.fr/hor/fdi:010050067 (consulté le 17 décembre 2019).

Sayad A., Dupuy E., 1995, Un Nanterre algérien : terre de bidonvilles, Paris, Éditions Autrement.

Simon P., 1996, Les immigrés et le logement : une singularité qui s'atténue, Données sociales, Institut national de la statistique et des études économiques, p. 421-428.

Thiery N., 2010, Identité parentale et insertion sociale en CHRS : le rôle de l'enfant accompagnant sa mère, Les Sciences de l'éducation - Pour l'Ère nouvelle, vol. 43, p. 81-100.

Thiery N., 2008, L'hébergement de femmes accompagnées d'enfants en CHRS : quelle incidence sur l'identité parentale ? Sociétés et jeunesses en difficulté [En ligne], n5 | Printemps 2008, mis en ligne le 21 juillet 2008 : http:// journals.openedition.org/sejed/2992 (consulté le 17 décembre 2019).

Revue des politiques sociales et familiales $\mathrm{n}^{\circ} 134-1^{\text {er }}$ trimestre 2020

92 Synthèses et statistiques 